TITLE:

\title{
Finite beta effects on MHD equilibria and energetic ion losses in a rippled tokamak
}

$\operatorname{AUTHOR}(S)$ :

Bunno, M.; Nakamura, Y.; Suzuki, Y.; Shinohara, K.; Matsunaga, G.

\section{CITATION:}

Bunno, M....[et al]. Finite beta effects on MHD equilibria and energetic ion losses in a rippled tokamak. Nuclear Fusion 2012, 52(8): 83009.

ISSUE DATE:

2012-07-11

URL:

http://hdl.handle.net/2433/160281

\section{RIGHT:}

(c) 2012 IAEA, Vienna; この論文は出版社版でありません。引用の際には 出版社版をご確認ご利用ください。; This is not the published version. Please cite only the published version. 


\title{
Finite beta effects on MHD equilibria and energetic ion losses in a rippled tokamak
}

\author{
M.Bunno $^{1}$, Y.Nakamura ${ }^{1}$, Y.Suzuki ${ }^{2}$, K.Shinohara ${ }^{3}$ and \\ G.Matsunaga ${ }^{3}$ \\ ${ }^{1}$ Graduate School of Energy Science, Kyoto University, Gokasho, Uji, Kyoto \\ 611-0011, Japan \\ 2 National Institute for Fusion Science, Toki, 509-5292, Japan \\ 3 Japan Atomic Energy Agency, Naka, Ibaraki 311-0193, Japan \\ E-mail: bunno.michinao.44v@st.kyoto-u.ac.jp
}

\begin{abstract}
The efficiency of energetic ion confinement is reduced by ripple fields, which are mainly generated by the finite number of toroidal field coils in a tokamak reactor. Finite beta effects alter magnetic field structures and energetic ion orbits. Finite beta effects on magnetohydrodynamic (MHD) equilibria have been thoroughly researched. However, it is not known how finite beta effects affect energetic ion losses. To investigate this, the MHD equilibrium was calculated using the VMEC code. Finite beta effects on the ripple ratio were accurately investigated and it was found that the non-axisymmetric poloidal field generated by the plasma current could not be ignored. The guiding center orbit equation was solved to clarify physical considerations of finite beta effects on the energetic ion losses. The energetic ion orbits were found to be strongly affected by the diamagnetic effect. By performing calculations for energetic ions with the same initial position, it was found that the diamagnetic effect produced two opposing effects: it reduced particle loss due to the closed $|B|$ contour and it increased particle loss due to the high curvature of the $|B|$ contour.
\end{abstract}




\section{Introduction}

Tokamaks are usually considered to be axisymmetric systems. However, the finite number of toroidal field coils (TFCs) produces ripples in the toroidal field that break the symmetry about the toroidal angle $\phi$. This non-axisymmetric field is called toroidal field (TF) ripples. The orbits of energetic ions such as alpha particles and neutral beam injection (NBI) ions are strongly affected by TF ripples [1][2][3]. These orbit losses cause several serious problems in tokamak reactors; for example, they reduce the plasma heating efficiency and the heat load concentration [4][5]. Accurate knowledge of the magnetic field inside and outside the plasma is required to analyze these problems. Since the plasma current also alters the field structures, magnetohydrodynamic (MHD) equilibrium equations must be solved. Since a high beta plasma is required to realize practical use of nuclear fusion reactors and improve their economic efficiency, finite beta effects on ripple-induced orbit losses need to be discussed for high beta plasmas.

The conventional procedure for studying finite beta effects on ripple-induced orbit losses consists of two stages: calculating the MHD equilibrium and calculating the orbit of energetic ions. The Grad-Shafranov equation is an axisymmetric MHD equilibrium equation. It has been applied to tokamaks by assuming them to be axisymmetric. The resulting equilibrium field is thus axisymmetric. However, the three-dimensional (3D) magnetic field can be approximated by superimposing the non-axisymmetric components of the vacuum field. This approximation method is known as the vacuum approximation and it has been conventionally used to analyze energetic ion losses in tokamak plasmas. Suzuki used the 3D MHD equilibrium equation code VMEC [6][7] to investigate finite beta effects on TF ripples [8]. They investigated in a relatively high beta tokamak plasma, $\langle\beta\rangle \sim 12.9 \%$, with an impractical circular cross-section. They clarified several finite beta effects, including the non-axisymmetric effect, the Shafranov shift, and the diamagnetic effect on deeply ripple trapped particles. However, since they did not calculate energetic ion orbits, finite beta effects on them were not discussed. Strumberger et al. [9] and Spong et al. [10] compared the 3D equilibrium fields obtained using the VMEC code with results obtained using the vacuum approximation and concluded that non-axisymmetric finite beta effects are negligibly small. However, those studies did not sufficiently discuss other finite beta effects on MHD equilibria and physical considerations of these effects. Furthermore, energetic ion losses were calculated only for the vacuum approximation in a low beta plasma.

Both MHD equilibrium and orbit calculations are required to precisely analyze finite beta effects on ripple-induced orbit losses. In the present study, the VMEC and GCB codes [11] are respectively used to perform MHD equilibrium and orbit calculations in a D-shaped tokamak; this is almost the same model as that used in Refs. [9] and [10]. The guiding center orbit equation can be calculated using the GCB code in Boozer coordinates from results obtained using the VMEC code [12][13][14]. To clarify the physical mechanism of finite beta effects, typical energetic ion orbits are calculated for various beta plasmas. Therefore, the actual loss rate of fusion alpha particles could not 
Table 1. Calculation parameters

\begin{tabular}{|r||c|c|c|c|c|}
\hline Scenario & $\langle\beta\rangle \%$ & $\beta_{\mathrm{N}}[\% \mathrm{mT} / \mathrm{MA}]$ & $\beta_{\mathrm{p}}$ & $\Psi_{\text {edge }}[\mathrm{Wb}]$ & $J_{\mathrm{p}}[\mathrm{MA}]$ \\
\hline $\mathrm{J} 1$ & 2.34 & 3.01 & 2.15 & 103.0 & 8.84 \\
\hline $\mathrm{J} 2$ & 3.99 & 5.19 & 3.54 & 99.9 & 9.17 \\
\hline $\mathrm{J} 3$ & 5.22 & 6.77 & 4.47 & 98.8 & 9.47 \\
\hline
\end{tabular}

be calculated in this study. The remainder of the paper is organized as follows. Finite beta effects on MHD equilibria are clarified for a realistic D-shaped rippled tokamak in Sec. 2. These effects on ripple-induced orbit losses are discussed in Sec. 3. Finally, Sec. 4 summarizes the findings and conclusions of this study.

\section{MHD equilibrium calculation}

MHD equilibria are calculated using the VMEC code, which is based on the energy principle expressed in flux coordinates $(s, \theta, \zeta)$, where $s$ is the normalized toroidal flux, $\theta$ is the poloidal angle, and $\zeta$ is the toroidal angle. The 18 TFCs generate a nonaxisymmetric vacuum field. Any ferrite materials are ignored in this study. The TFC positions have toroidal angles given by

$$
\phi=\frac{2 \pi}{18} k \quad(k=0,1, \ldots, 17) .
$$

Table 1 lists the calculation parameters, such as the volume-averaged beta value $\langle\beta\rangle$, the normalized beta $\beta_{\mathrm{N}}$, the poloidal beta $\beta_{\mathrm{p}}$, the toroidal magnetic flux at the plasma surface $\Psi_{\text {edge }}$, and the plasma current $J_{\mathrm{p}}$. A volume-averaged beta $\langle\beta\rangle$ of $5.22 \%$ for $\mathrm{J} 3$ is realistic for a spherical tokamak and the commercial reactor DEMO. To set the plasma inside the given separatrix, $\Psi_{\text {edge }}, J_{\mathrm{p}}$ and the external poloidal coil currents are changed. Figures 1(a) and (b) respectively show pressure $P$ profiles for three beta values and the safety factor profile $q$ as a function of the normalized toroidal flux. Because 18 TFCs produce $2 \pi / 18$ toroidal periods, the computational cost can be reduced by employing periodic boundary conditions. In the VMEC calculations, the number of radial grid points for $s$ is 40 and the poloidal and toroidal mode numbers are 12 and 8 , respectively.

\subsection{Finite beta effects on field structures}

This section clarifies finite beta effects on the field structures. There are two plasma currents in a fusion plasma: poloidal and toroidal currents. The poloidal current $j_{\mathrm{p}}$ is called the diamagnetic current because it reduces the toroidal field in a high beta plasma $\beta_{\mathrm{p}}>1$. Figures $2(\mathrm{a})$ and (b) respectively show the toroidal and vertical field components of J3 along the $R$ direction at $(Z, \phi)=(0.5 \mathrm{~m}, 0)$. The red, green, and blue lines in this figure indicate the vacuum field due to the external coil current, the field due to the plasma current, and the equilibrium field, respectively. At $R=7 \mathrm{~m}$, the 

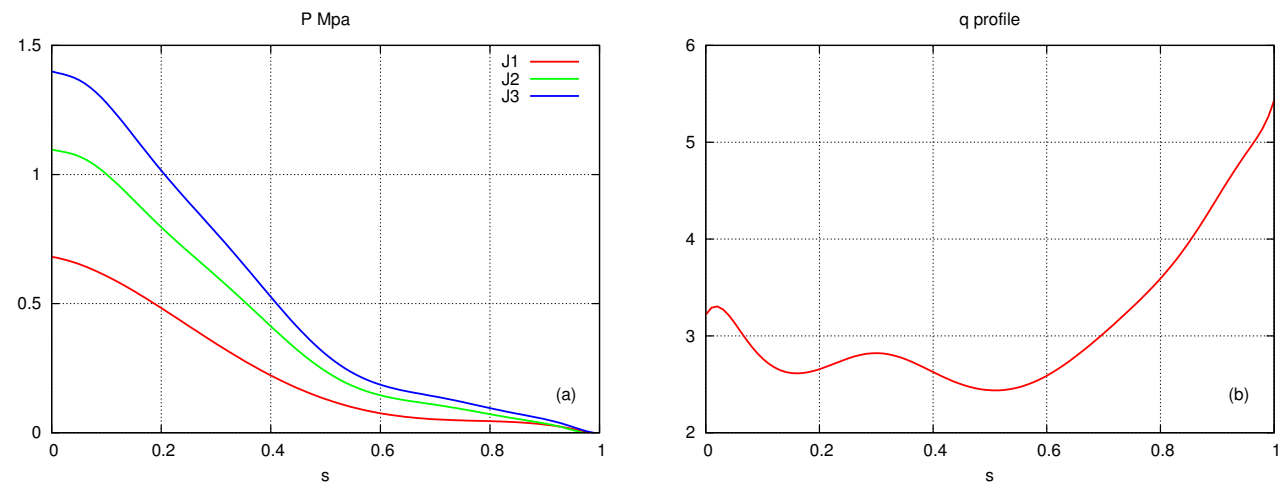

Figure 1. (a) Pressure $P$ and (b) safety factor $q$ as functions of normalized toroidal flux $s$

toroidal field is strongly reduced to $4.5 \mathrm{~T}$ from $4.7 \mathrm{~T}$, which is a $4 \%$ reduction. Since a huge toroidal current $j_{\mathrm{t}}$ is required to generate closed flux surfaces in a tokamak plasma, it is usually larger than $j_{\mathrm{p}}$. The magnitude of $B_{z}$ is high, especially at the outer torus. Figure 3 shows the magnitude of the equilibrium toroidal field $B_{\mathrm{t}}$, the vacuum field $\left|B_{\text {vac }}\right|$, and the equilibrium field $\left|B_{\text {equ }}\right|$ for J3 in the $R$ direction at $(Z, \phi)=(0.5 \mathrm{~m}, 0)$. The vacuum field strength $\left|B_{\text {vac }}\right|$ is inversely proportional to $R$. At $R=7.5 \mathrm{~m},\left|B_{\text {equ }}\right|$ is reduced by the diamagnetic effect, while it is increased by the poloidal field due to the plasma current beyond $R=7.5 \mathrm{~m}$. In the results, $\left|B_{\text {equ }}\right|$ has the same value at the same $Z$ position and there is a hole in the $R$ direction. This causes the $\left|B_{\text {equ }}\right|$ contour to be closed at the outer torus; this effect is discussed in Sec. 2.4.

To accurately investigate finite beta effects, the non-axisymmetric field component is represented according to [8],

$$
\begin{aligned}
\Delta B & \equiv B(\phi=0)-B(\phi=\pi / 18) \\
& \sim \frac{\Delta B_{\mathrm{t}}^{\prime}+\Delta B_{\mathrm{t}}^{*}}{\sqrt{1+\left(\left\langle B_{\mathrm{p}}\right\rangle /\left\langle B_{\mathrm{t}}\right\rangle\right)^{2}}}+\frac{\Delta B_{\mathrm{p}}^{\prime}+\Delta B_{\mathrm{p}}^{*}}{\sqrt{1+\left(\left\langle B_{\mathrm{t}}\right\rangle /\left\langle B_{\mathrm{p}}\right\rangle\right)^{2}}},
\end{aligned}
$$

where $\langle B\rangle$ is the axisymmetric field component, $B^{\prime}$ is the vacuum field, and $B^{*}$ is the field generated by the plasma current. Unlike [8], $\Delta B$ includes the non-axisymmetric poloidal field. This gives rise to the following equation

$$
\Delta B \sim \Delta B_{\mathrm{t}}^{\prime}-\frac{1}{2}\left(\frac{\left\langle B_{\mathrm{p}}\right\rangle}{\left\langle B_{\mathrm{t}}\right\rangle}\right)^{2} \Delta B_{\mathrm{t}}^{\prime}+\Delta B_{\mathrm{t}}^{*}+\frac{\left\langle B_{\mathrm{p}}\right\rangle}{\left\langle B_{\mathrm{t}}\right\rangle}\left(\Delta B_{\mathrm{p}}^{\prime}+\Delta B_{\mathrm{p}}^{*}\right) .
$$

$\Delta B$ is affected not only by the non-axisymmetric field but also by the axisymmetric toroidal and poloidal fields. $\Delta B_{\mathrm{t}}^{\prime}$ has the largest effect because it is induced by the TF ripple. In contrast, the effect of $\Delta B_{\mathrm{p}}^{\prime}$ is negligibly small. The magnitude of $\Delta B_{\mathrm{p}}^{*}$ is larger than that of $\Delta B_{\mathrm{t}}^{*}$ because $j_{\mathrm{t}}$ is larger than $j_{\mathrm{p}}$ in a tokamak plasma. However, the effect of the non-axisymmetric poloidal field is reduced by the factor $\left\langle B_{\mathrm{p}}\right\rangle /\left\langle B_{\mathrm{t}}\right\rangle$. Therefore, the effect of $\Delta B_{\mathrm{p}}^{*}$ becomes important at the outer torus.

The effects of $\Delta B_{\mathrm{t}}^{*}$ and $\Delta B_{\mathrm{p}}^{*}$ should be considered separately. According to [8], $j_{\mathrm{t}}$ is largest and $j_{\mathrm{p}}$ is smallest under the TFCs in the toroidal direction. Since the poloidal 

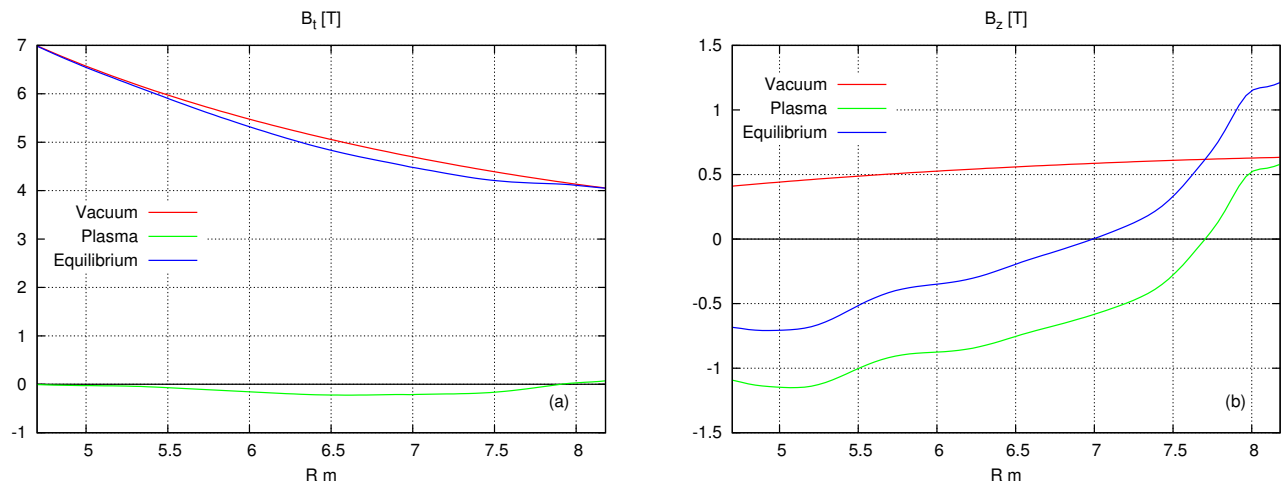

Figure 2. (a) $B_{\mathrm{t}}$ and (b) $B_{\mathrm{z}}$ of the vacuum field, the field generated by the plasma current, and the equilibrium field for $\mathrm{J} 3$ in the $R$ direction at $(Z, \phi)=(0.5 \mathrm{~m}, 0)$

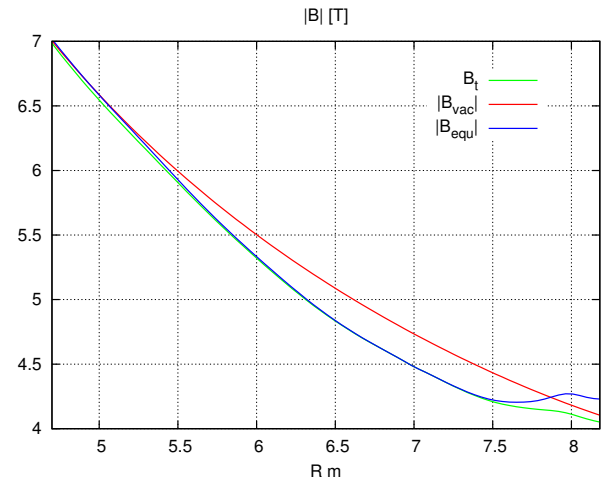

Figure 3. Magnitudes of the equilibrium toroidal field $B_{\mathrm{t}}$, the vacuum field $\left|B_{\mathrm{vac}}\right|$, and the equilibrium field $\left|B_{\text {equ }}\right|$ for $\mathrm{J} 3$ in the $R$ direction at $(Z, \phi)=(0.5 \mathrm{~m}, 0)$

field is mainly generated by $j_{\mathrm{t}}$, the poloidal field from $j_{\mathrm{t}}$ is largest and smallest at the top and bottom of the ripples, respectively. In the results, $j_{\mathrm{t}}$ mainly enhances the nonaxisymmetric field, whereas the toroidal field is mainly generated by the TFCs. If $j_{\mathrm{p}}$ is diamagnetic for $\beta_{\mathrm{p}}>1$ plasma, $B_{\mathrm{t}}$ will be reduced more at the bottom than at the top of the ripples, so $\Delta B_{\mathrm{t}}^{*}$ will be increased. This implies that the non-axisymmetric effects due to both $j_{\mathrm{t}}$ and $j_{\mathrm{p}}$ increase the ripple field in a high beta plasma.

\subsection{Ripple ratio along the toroidal angle}

The ripple ratio $\delta$ along the toroidal angle $\phi$ is defined by

$$
\delta=\frac{B_{\max }-B_{\min }}{B_{\max }+B_{\min }}
$$

where $B_{\max }$ and $B_{\min }$ are respectively the maximum and minimum field strengths $|B|$ along the toroidal angle. Strumberger et al. [9] and Spong et al. [10] used $\delta$ to evaluate the non-axisymmetric field. This allows us to easily analyze the non-axisymmetric finite beta effect. Figure 4(a) shows the ripple ratio for the vacuum toroidal ripple field $\delta_{\text {vac }}$ on 
Table 2. $\delta$ components at $(R, Z)=(7.9 \mathrm{~m}, 0.5 \mathrm{~m})$ for $\mathrm{J} 3$

\begin{tabular}{|r|c||c|c|c|c||c|}
\hline Scenario & $\langle\beta\rangle \%$ & $\delta_{\text {vac }}$ & $\delta_{2 \mathrm{D}}$ & $\delta_{\mathrm{t}}^{*}$ & $\delta_{\mathrm{p}}^{*}$ & $\delta$ \\
\hline J3 & \multirow{2}{*}{5.22} & 0.004909 & -0.000189 & 0.000138 & 0.000291 & 0.005149 \\
\cline { 3 - 7 } & & $100 \%$ & $-3.85 \%$ & $2.81 \%$ & $5.93 \%$ & $104.88 \%$ \\
\hline
\end{tabular}

the poloidal cross-section. At the outer torus, $\delta_{\mathrm{vac}}$ is high because the gap between the TFCs is greater so that the non-axisymmetric field generated by the TFCs is greater. The maximum value of $\delta_{\text {vac }}$ is almost 0.01 , which is considered to be the upper limit for tokamaks such as JT-60U based on consideration of energetic ion losses and plasma confinement [15]. The finite beta effects alter the ripple ratio. Figure 4(b) shows the ripple ratios $\delta$ for the vacuum ripple field $\delta_{\text {vac }}$ and for scenario J3, $\delta(\mathrm{J} 3)$. Figure $5(\mathrm{a})$ shows $\delta_{\text {vac }}$ and $\delta(\mathrm{J} 3)$ in the $R$ direction at $(Z, \phi)=(0.5 \mathrm{~m}, 0)$. In these figures, $\delta$ is lower at the outer torus and higher in the core region. To perform detailed analysis, the $\delta$ components are described by equation (3),

$$
\begin{aligned}
\delta & =\frac{B_{\max }-B_{\min }}{B_{\max }+B_{\min }}=\frac{\Delta B}{2\langle B\rangle} \\
& \sim \frac{1}{2\langle B\rangle}\left[\Delta B_{\mathrm{t}}^{\prime}-\frac{1}{2}\left(\frac{\left\langle B_{\mathrm{p}}\right\rangle}{\left\langle B_{\mathrm{t}}\right\rangle}\right)^{2} \Delta B_{\mathrm{t}}^{\prime}+\Delta B_{\mathrm{t}}^{*}+\frac{\left\langle B_{\mathrm{p}}\right\rangle}{\left\langle B_{\mathrm{t}}\right\rangle} \Delta B_{\mathrm{p}}^{*}\right] \\
& \sim \frac{\Delta B_{\mathrm{t}}^{\prime}}{2\left\langle B_{\mathrm{t}}^{\prime}\right\rangle}-\left(\frac{\left\langle B_{\mathrm{t}}^{\prime}\right\rangle\left\langle B_{\mathrm{t}}^{*}\right\rangle+\left\langle B_{\mathrm{p}}\right\rangle^{2}}{2\left\langle B_{\mathrm{t}}\right\rangle^{3}}\right) \Delta B_{\mathrm{t}}^{\prime}+\frac{\Delta B_{\mathrm{t}}^{*}}{2\left\langle B_{\mathrm{t}}\right\rangle}+\left(\frac{\left\langle B_{\mathrm{p}}\right\rangle}{\left\langle B_{\mathrm{t}}\right\rangle}\right) \frac{\Delta B_{\mathrm{p}}^{*}}{2\left\langle B_{\mathrm{t}}\right\rangle} \\
& \sim \delta_{\mathrm{vac}}+\delta_{2 \mathrm{D}}+\delta_{\mathrm{t}}^{*}+\delta_{\mathrm{p}}^{*},
\end{aligned}
$$

where $\delta_{2 \mathrm{D}}, \delta_{\mathrm{t}}^{*}$, and $\delta_{\mathrm{p}}^{*}$ are the finite beta effects of the axisymmetric component and the non-axisymmetric toroidal and poloidal components, respectively. To clarify the finite beta effect on the TF ripple, Fig. 5(b) shows each $\delta$ component for J3 along the $R$ direction at $(Z, \phi)=(0.5 \mathrm{~m}, 0)$. While all the finite beta effects are comparable, they are much smaller than $\delta_{\text {vac }}$.

Table 2 shows each $\delta$ component at $(R, Z)=(7.9 \mathrm{~m}, 0.5 \mathrm{~m})$ for J3. The axisymmetric finite beta effects $\delta_{2 \mathrm{D}}$ reduce the vacuum toroidal ripple $\delta_{\text {vac }}$ by $3.85 \%$, whereas the non-axisymmetric finite beta effects $\delta_{\mathrm{t}}^{*}$ and $\delta_{\mathrm{p}}^{*}$ increase $\delta_{\mathrm{vac}}$ by 2.81 and $5.93 \%$, respectively. This means that $\delta$ of the 3D MHD equilibrium field is increased by $8.73 \%$ compared to $\delta$ for the vacuum approximation in the $\langle\beta\rangle=5.22 \%$ plasma. We were unable to determine whether this $8.73 \%$ increase in $\delta$ is negligibly small; we intend to investigate this problem by calculating the actual loss rate of the energetic ions in a future study.

\subsection{Ripple well depth along magnetic field line}

In the previous section, the non-axisymmetric field was evaluated as a function of the toroidal angle for fixed $R$ and $Z$. However, for energetic ion orbits, it is more meaningful 

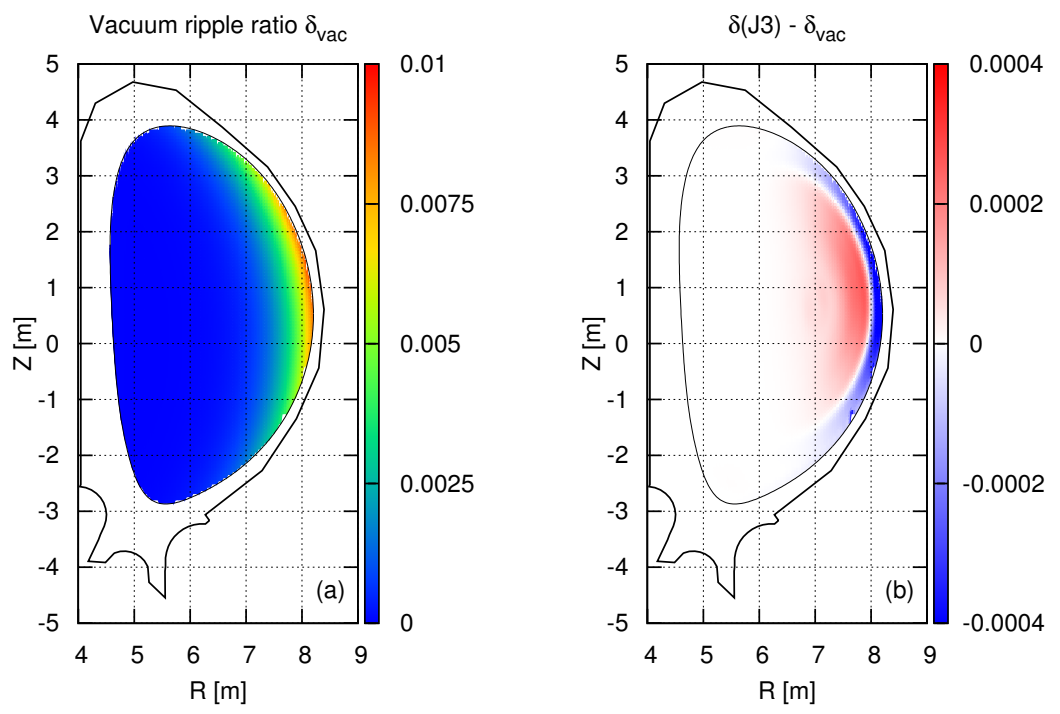

Figure 4. Distributions of $\delta$ for (a) the vacuum toroidal ripple field and (b) J3 on the poloidal cross-section
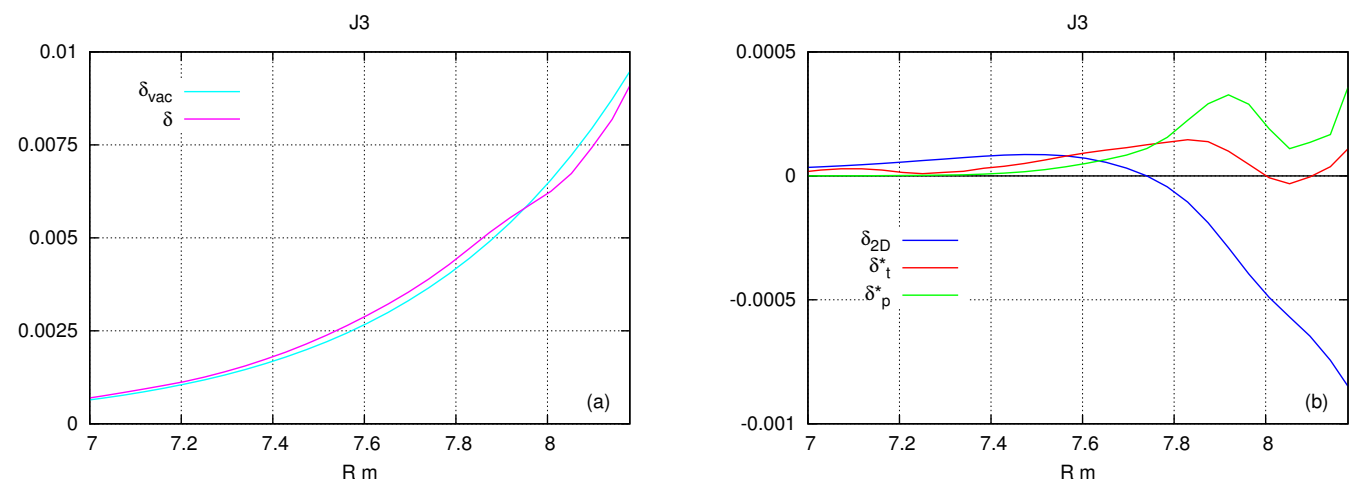

Figure 5. (a) Vacuum toroidal ripple field and ripple field for $\mathrm{J} 3$ and (b) each $\delta$ component for $\mathrm{J} 3$ in the $R$ direction at $(Z, \phi)=(0.5 \mathrm{~m}, 0)$

to define the ripple field along the field line. The ripple well depth $d_{w}$ along the field line is defined by

$$
\mathrm{d}_{w}=\frac{B_{\max }-B_{\min }}{B_{\max }+B_{\min }}
$$

where $B_{\max }$ and $B_{\min }$ are respectively the maximum and minimum values of $|B|$ along the field line. Figure $6(\mathrm{a})$ shows the $\mathrm{d}_{w}$ distribution for $\mathrm{J} 3$ on the poloidal cross-section. The ripple well depth $\mathrm{d}_{w}$ depends strongly on the field line pitch (i.e., the local inclination of the field lines). The ripple well exists if the following condition is satisfied

$$
\alpha \equiv\left|\frac{(\partial \bar{B} / \partial l)}{(\partial \tilde{B} / \partial l)}\right|<1
$$



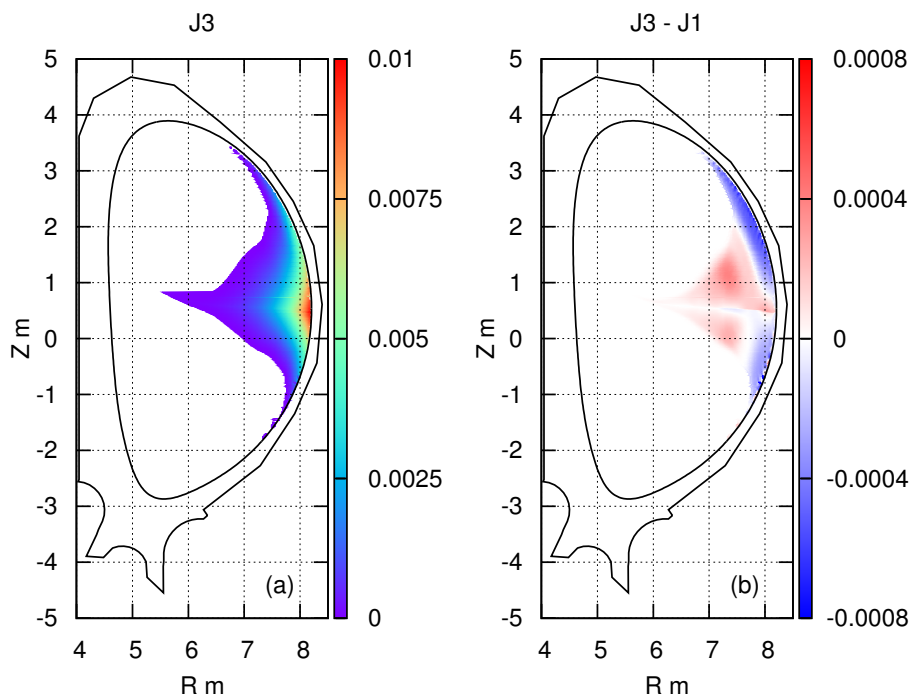

Figure 6. Distribution of $\mathrm{d}_{w}$ for (a) $\mathrm{J} 3$ and (b) $\mathrm{J} 3-\mathrm{J} 1$ in the poloidal cross-section

where $\bar{B}$ is the axisymmetric field, $\tilde{B}$ is the non-axisymmetric field, and $l$ is the distance along the field line [16][17]. For a steeper pitch, $\alpha$ is increased and $\mathrm{d}_{w}$ is reduced. Figure 6(b) shows the difference in the ripple well depths $\mathrm{d}_{w}$ for J3 and J1 (i.e., J3 - J1) to clarify the finite beta effect on the ripple well. In this figure, $\delta$ is reduced at the outer torus and increased at the core region. This relation is more remarkable than $\delta$ in Sec. 2.2. It implies that the field line pitch is steep in a high beta plasma at the outer torus.

The Pfirsch-Schlüter current flows along the magnetic field line. This parallel current generates the poloidal magnetic field and alters the field line pitch. Figure 7(a) shows $|B| /\left|B_{0}\right|$ on the field line that passes through $(R, Z, \phi)=(8.0 \mathrm{~m}, 0.5 \mathrm{~m}, 0)$ for the $2 \mathrm{D}$ equilibrium field, where $B_{0}$ is $|B|$ at $(R, Z, \phi)=(8.0 \mathrm{~m}, 0.5 \mathrm{~m}, 0)$. As mentioned above, the field line pitch becomes steeper at the outer torus with increasing beta value. Figure 7(b) shows $|B| /\left|B_{0}\right|$ for the 3D equilibrium field. In this figure, $\mathrm{d}_{w}$ is reduced at the outer torus because of the steeper field line pitch in a high beta plasma.

\subsection{Change in $B_{\min }$ contour}

The deeply ripple-trapped particles are repeatedly reflected by the magnetic mirror effect and they drift along the bottom of the ripple well [18]. The $B_{\min }$ contour, which was defined in Sec. 2.2, can be used to estimate and control their orbits.

Figures $8(\mathrm{a}),(\mathrm{b})$, and (c) show the $B_{\text {min }}$ contours for J1, J2, and J3, respectively. At the outer torus, the contour curvature becomes higher with increasing beta value and it eventually becomes closed at the outer torus. This phenomena is induced by the diamagnetic effect of $j_{\mathrm{p}}$ and the paramagnetic effect of $j_{\mathrm{t}}$, as mentioned in Sec. 2.1. The curved $B_{\min }$ contour enables the deeply ripple-trapped particles to move more easily to high $\delta$ and $\mathrm{d}_{w}$ regions. However, if this contour is completely closed in a high beta 

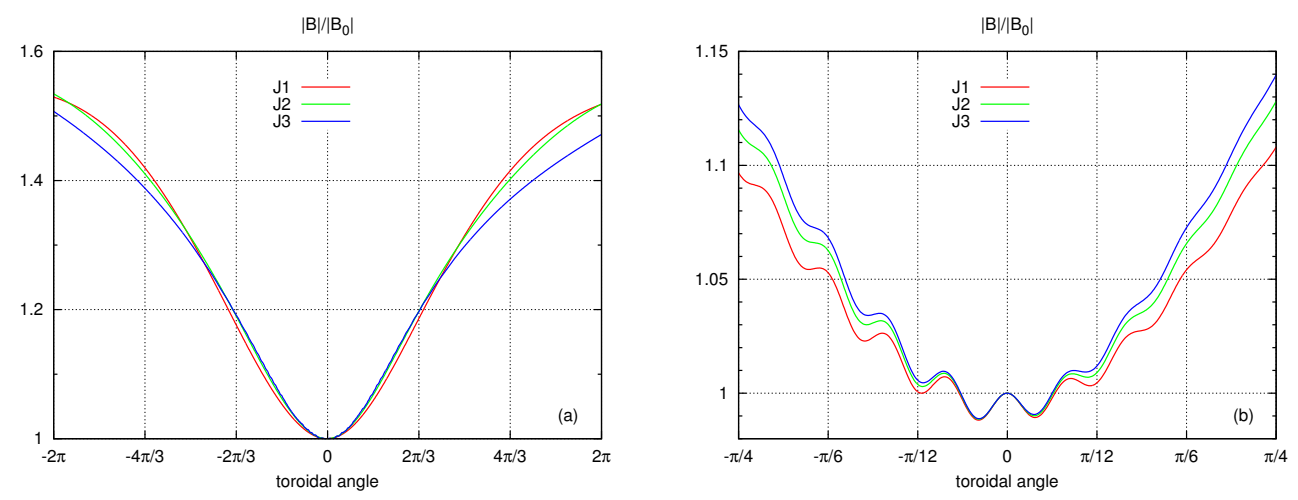

Figure 7. Variation in $|B| /\left|B_{0}\right|$ for (a) 2D and (b) 3D MHD equilibrium field on the field line that passes through $(R, Z, \phi)=(8.0 \mathrm{~m}, 0.5 \mathrm{~m}, 0)$

plasma, the trapped particle losses will be reduced.

Not only the deeply ripple-trapped particles, but also the banana-trapped particles are associated with the $|B|$ contour because the banana tip also moves along it. Because the parallel velocity $v_{\|}$is zero at the banana tip, the magnetic moment $\mu_{m}$ can be written as

$$
\mu_{m}=\frac{m v_{\perp}^{2}}{2|B|}=\frac{m v^{2}}{2 B_{\mathrm{tip}}},
$$

where $B_{\text {tip }}$ is $|B|$ at the banana tip. The banana tip follows the $|B|$ contour because $\mu_{m}$ and $\frac{m v^{2}}{2}$ are conserved. If the particles are trapped in the closed $|B|$ contour region, they will not be lost. However, if the particles are trapped in the non-closed $|B|$ contour region, their orbits will strongly depend on the $|B|$ contour curvature. The finite beta effect increases the $|B|$ contour curvature and the banana tip also more easily move to the outer torus. Therefore, the particles, which are trapped in the non-closed $|B|$ contour region, are more strongly affected by the ripple field and the number of loss particles will be increased. This is confirmed in Section 3 where their orbits are calculated.

\subsection{Change in the flux surface positions}

The Shafranov shift strongly affects the alpha particle loss, which are produced by a thermal-thermal reaction. The vertical field is generated by the Pfirsch-Schlüter current and the flux surfaces are shifted to the outer torus. Figure 9 shows the changes in the positions of the normalized toroidal flux surfaces $s=(0.2,0.4,0.6,0.8$, and 1.0) at $\zeta=0$.

The Shafranov shift moves the generation points of the alpha particles out to the outer torus, namely the higher ripple region. In addition, it reduces the distance from the generation point to the first wall. In the results, the Shafranov shift increases the number of loss particles and the heat loads from the fusion alpha particles. 

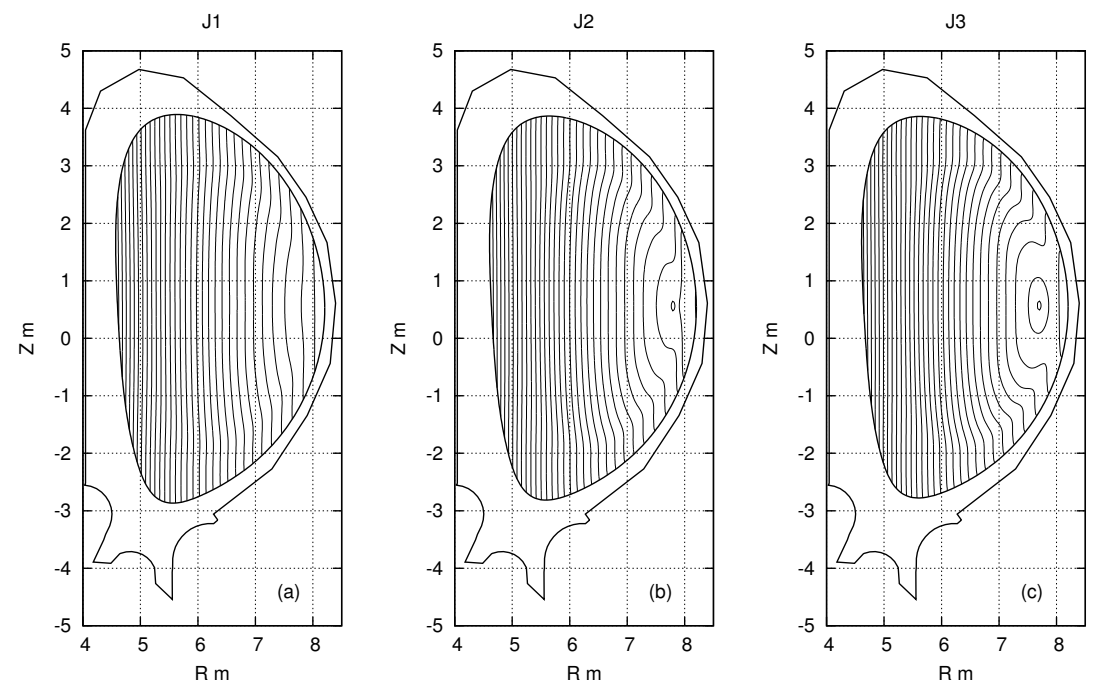

Figure 8. $B_{\min }$ contours for (a) J1, (b) J2, and (c) J3 in the poloidal cross-section

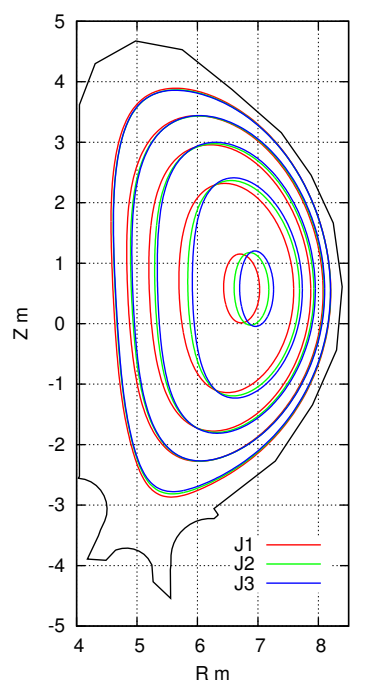

Figure 9. Changes in the position of $s=(0.2,0.4,0.6,0.8$, and 1.0) at $\zeta=0$ for each beta value

\section{Finite beta effects on energetic ion orbits}

\subsection{Classification of orbits in a rippled tokamak}

The finite beta effects on the MHD equilibria were clarified in the previous section. However, orbit calculation is also required to quantitatively estimate the finite beta effects on the energetic ion losses. In this study, the energetic ion orbits are calculated by the GCB code, which is based on the guiding center orbit equations in Boozer coordinates. However, the orbits cannot be determined outside the plasma because flux coordinates are used. This calculation also ignores collisions. Therefore, the particles 
are assumed to be loss when the guiding center reaches the plasma boundary, unless the tracing time exceeds $0.01 \mathrm{~s}$, in which case they are assumed to be non-loss particles. While the actual loss rate of energetic ions could not be obtained, it is sufficient to only classify the orbits in a rippled tokamak.

Figures 10(a), (b), and (c) show the classification of alpha particles orbits that start at $(R, Z, \phi)=(7.5 \mathrm{~m}, 0.5 \mathrm{~m}, \pi / 18)$ for $\mathrm{J} 1, \mathrm{~J} 2$, and $\mathrm{J} 3$, respectively. The initial energy $E_{0}$ is divided between 1.0 and $3.5 \mathrm{MeV}$ in $0.1 \mathrm{MeV}$ intervals and the pitch angle $\lambda$, which is defined as

$$
\lambda=\frac{1}{\pi} \operatorname{Tan}^{-1}\left(\frac{v_{\perp}}{v_{\|}}\right) .
$$

is divided between 0 and 1 in 0.01 intervals. They are classified according to the following colors:

- Passing particles [Blue]

- Banana-trapped loss particles [Red]

- Banana-trapped non-loss particles [Light blue]

- Ripple-trapped loss particles [Black]

- Ripple-trapped non-loss particles [Yellow],

where ripple-trapped particles are defined as trapped particles that are confined only in the $\mathrm{d}_{w}$ region and banana-trapped particles are the other trapped particles. Figures 11(a), (b), and (c) show the $B_{\min }$ contours (black line) and orbits (red line) of the particles, which start from $(R, Z, \phi)=(7.5 \mathrm{~m}, 0.5 \mathrm{~m}, \pi / 18)$ for $\mathrm{J} 1$, J2, and J3, respectively. For J3, the $B_{\min }$ contour is closed at the initial point; it thus describes a closed path. For J1 and J2, these particles reach the plasma boundary. For J1, the deeply ripple-trapped particles escape from the $\mathrm{d}_{w}$ region and become disturbed banana particles. However for J2, the orbit follows the $B_{\min }$ contour because this contour curves toward the high ripple region.

Figures 12(a) and (b) respectively show the loss fraction $\epsilon$ and the loss position on the plasma boundary for $3.5 \mathrm{MeV}$ alpha particles with respect to $\lambda$. The initial position is fixed at $(R, Z, \phi)=(7.5 \mathrm{~m}, 0.5 \mathrm{~m}, \pi / 18)$ and $\lambda$ is divided between 0 and 1 in 0.0001 intervals. Figure 13(a) shows that the loss process can be separated into rapid and slow loss. The rapid loss particles appear only in J2; they thus originate from deeply ripple-trapped loss particles. Furthermore, the localized loss position appears only for J2 in Fig. 13(b) around $(Z, \phi)=(-0.8 \mathrm{~m}, \pi / 36)$. Banana-trapped loss particles have a longer loss time than deeply ripple-trapped particles and the loss positions of bananatrapped loss particles are widely distributed over the entire plasma boundary. However, the condition for deeply ripple-trapped particles is strict, namely $\lambda \sim 0.5$ at the bottom of the ripple well (in this study, $\phi=\pi / 18$ ). The deeply ripple-trapped and bananatrapped loss particles have quite different loss times and positions. Therefore, the two loss processes should be distinguished when analyzing finite beta effects on energetic ion losses. 

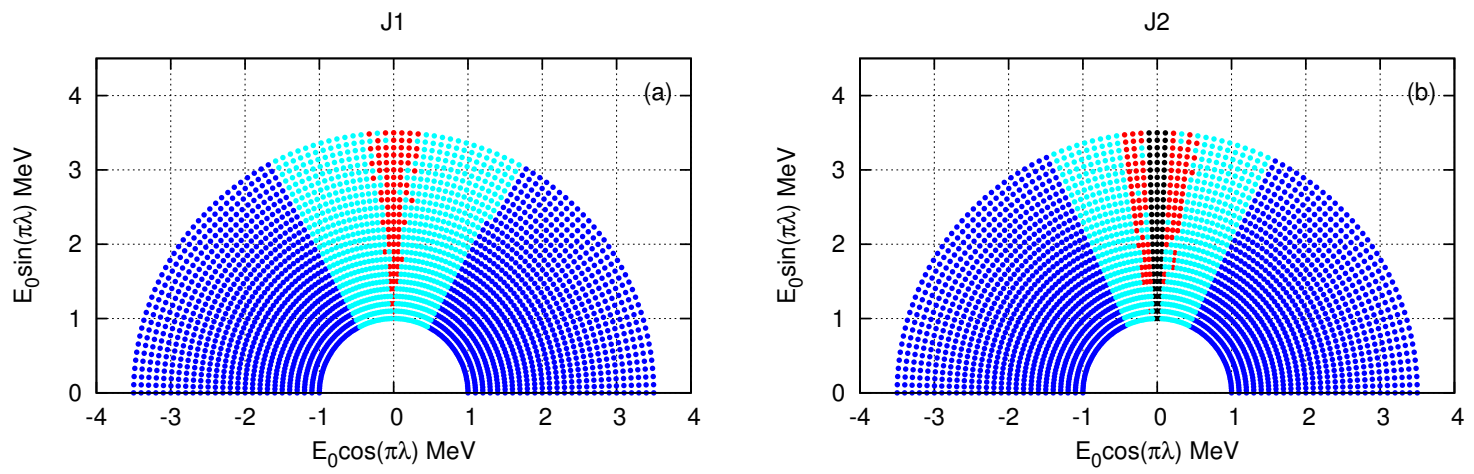

J3

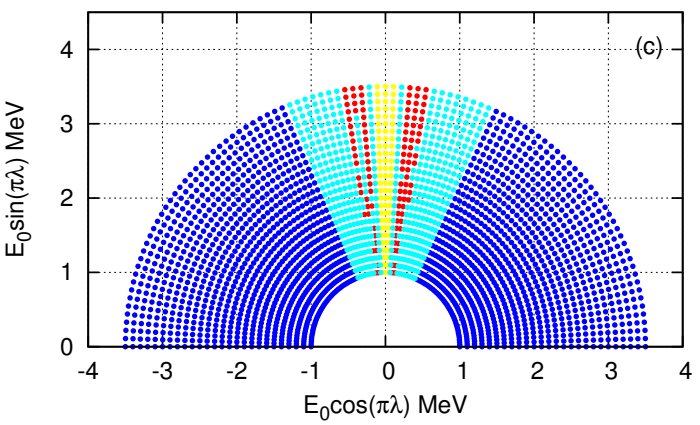

Figure 10. Classification of alpha particles starting from $(R, Z, \phi)=$ (7.5 m, $0.5 \mathrm{~m}, \pi / 18$ ) for (a) J1, (b) J2, and (c) J3
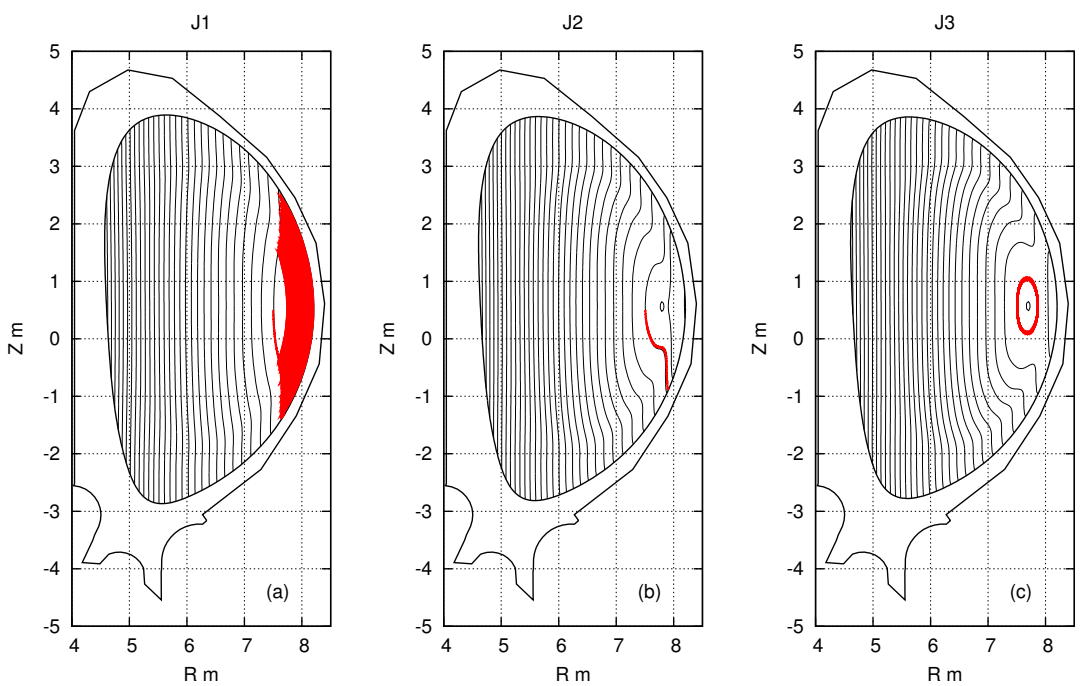

Figure 11. $B_{\min }$ contour (black line) and orbits of the particles (red line) that start from $(R, Z, \phi)=(7.5 \mathrm{~m}, 0.5 \mathrm{~m}, \pi / 18)$ with $\lambda=0.5$ for (a) J1, (b) J2, and (c) J3 

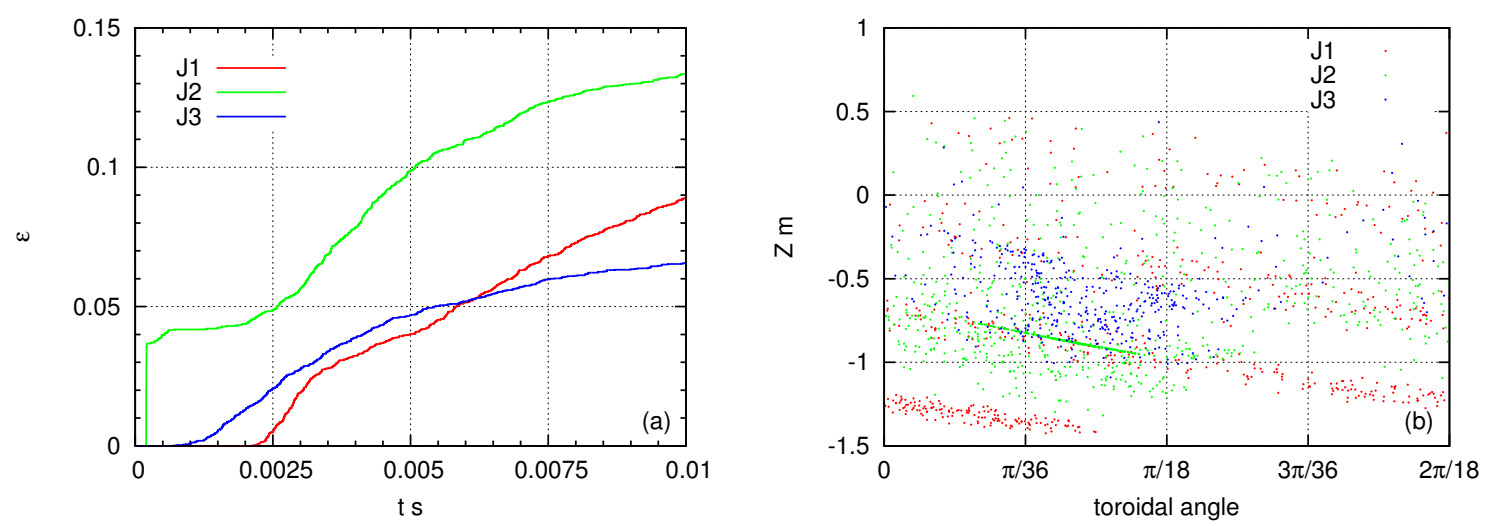

Figure 12. (a) Loss fraction $\epsilon$ and (b) loss position of alpha particles with respect to $\lambda$, which start from $(R, Z, \phi)=(7.5 \mathrm{~m}, 0.5 \mathrm{~m}, \pi / 18)$

\subsection{Finite beta effects on trapped particles}

In the previous section, energetic ion orbits were categorized with respect to $\lambda$. In this section, the orbits of trapped particles are analyzed with respect to the initial position. Figures 13(a), (b), and (c) show the classification of the trapped particles that start from $\phi=\pi / 18$ with $\lambda=0.5$, using the same color scheme as Fig. 10.

For J2, the number of ripple-trapped particles (black region) is increased at the outer torus for two reasons: the increased non-axisymmetric field at the core region and the curved $B_{\min }$ contour, as mentioned in Sec. 2.4. The latter is the more important effect because the boundary between the red and black regions has a similar shape to that of the $B_{\min }$ contour and the increase in the ripple ratio is quite small inside the plasma. The closed $B_{\min }$ contour region (the yellow region) for J2 is quite small, whereas the yellow region becomes remarkable for J3 and the number of deeply rippletrapped loss particles is highly reduced by this effect. The number of banana-trapped loss particles (the red region) is increased for $\mathrm{J} 3$. At $Z=0.5 \mathrm{~m}$, the boundary between the red and light-blue region is located at $7 \mathrm{~m}$ for $\mathrm{J} 1$ and $6.6 \mathrm{~m}$ for J3. Banana-trapped loss particles also originate from the curved $B_{\min }$ contour. A yellow region appears near $(R, Z)=(5 \mathrm{~m}, 1 \mathrm{~m})$. At the inner torus, there are strong ripples because the field strength along the field line is a maximum and the numerator of equation (7) becomes quite small. Therefore, particles starting from $(R, Z)=(5 \mathrm{~m}, 1 \mathrm{~m})$ with $\lambda=0.5$ will be trapped by the ripple well and become passing particles. In the results, they are categorized as ripple-trapped non-loss particles.

In these figures, the magnitude of the finite beta effects strongly depends on the generation points of the targeted energetic ions. For example, the positions and the pitch angle of alpha particles produced by the thermal-thermal reaction are randomly disturbed. Therefore, the number of deeply ripple-trapped particles is quite low. However, for NBI-generated fast ions, the strong confinement of deeply trapped particles permits quasi-perpendicular injection to realize better beam penetration. In 

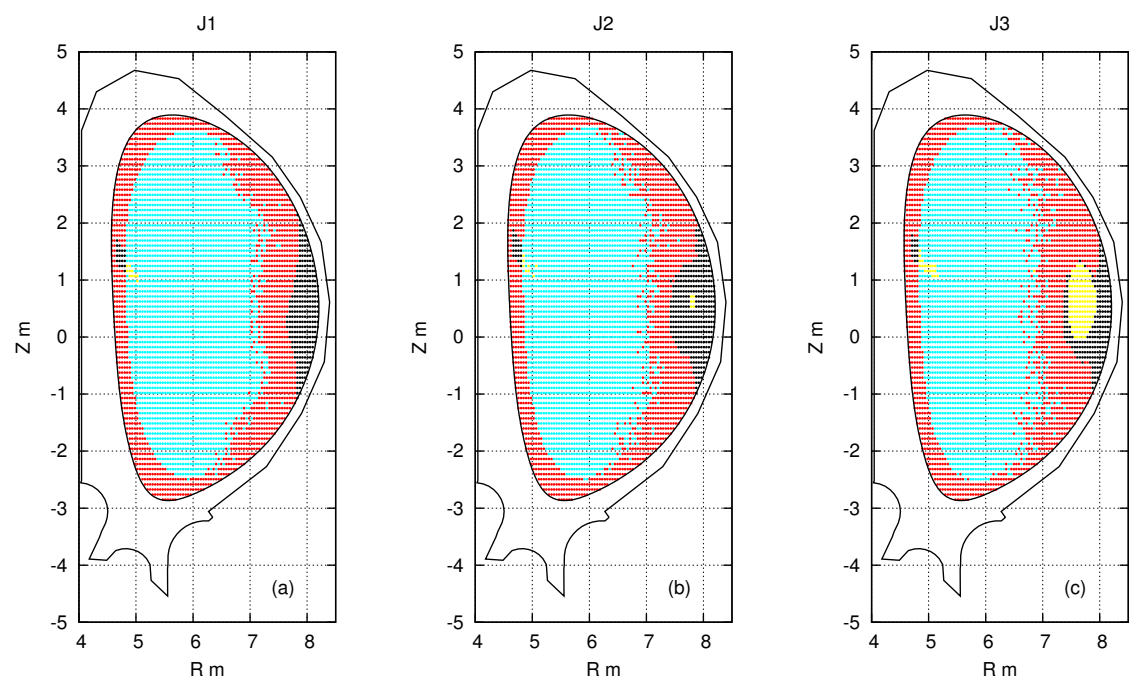

Figure 13. Classification of trapped particles that start from $\phi=\pi / 18$ with $\lambda=0.5$ for (a) J1, (b) J2, and (c) J3

the future, we intend to statistically investigate ripple-induced orbit losses by Monte Carlo calculations while considering birth profiles using codes such as the F3D-OFMC code [19].

\section{Summary}

Finite beta effects on energetic ion losses were studied in a realistic D-shaped tokamak. The finite beta effects on MHD equilibria were clarified. This topic has been investigated by Suzuki et al. [8] and Strumberger et al. [9]. Suzuki et al. state that "the magnitude of $\delta B_{\mathrm{P}}$ is usually very small compared with $\delta B_{\mathrm{T}}$ ". However, the opposite relation was observed in this study, which indicates that the non-axisymmetric poloidal field cannot be ignored. Strumberger et al. mainly focused on non-axisymmetric finite beta effect. In their calculations, the non-axisymmetric finite beta effect increased the ripple ratio by $8.2 \%$, which is very similar to the $8.73 \%$ increase obtained in the present study. To precisely clarify the physical considerations, the finite beta effects on the ripple ratio were categorized using equation (5). The non-axisymmetric toroidal and poloidal fields generated by the plasma current respectively increased the ripple ratio by up to 2.81 and $5.93 \%$ and the axisymmetric finite beta effect reduced it by $3.85 \%$.

Suzuki et al. said the $B_{\min }$ contour was closed in an unrealistic high beta plasma [8]. In this study, the $B_{\text {min }}$ contour started to be closed for $\langle\beta\rangle=4 \%$ and a wide closed $|B|$ contour region was obtained for $\langle\beta\rangle=5 \%$. Unlike [8], we investigated how much these finite beta effects alter the energetic ion orbits. The orbit calculations were performed from the same initial position $(R, Z)$ in a different beta plasma. In Fig. 13, a non-loss particle (yellow) region appears at the outer torus for J2 and J3. The diamagnetic effect reduces the loss particles, which are trapped in the closed $|B|$ contour 
region. If the particles are trapped in the non-closed $|B|$ contour region, their orbits will strongly depend on the contour curvature. Since the $|B|$ contour curvature is increased by the diamagnetic effect, the trapped particles can easily move to the outer torus (i.e., the high ripple region). The banana-trapped loss (red) region extends inside the plasma with increasing beta value. This implies that other trapped particles inside the plasma become loss particles by finite beta effects. This effect has not been mentioned in previous studies; we clarified it by calculating the energetic ion orbits.

The magnitude of the finite beta effects depends strongly on the generation points of the targeted energetic ions. In the future, we will attempt to estimate the actual loss rate of energetic ions such as alpha particles and NBI-generated fast ions including the birth profiles and Coulomb collisions using the F3D-OFMC code [19]. Quantitative evaluation of finite beta effects on orbit losses for the target energetic ions will greatly facilitate designing reactors that strongly confine energetic ions. Additional calculations are required to realize this. It is not only important to change the beta value, but it is also important to compare with other field structures such as the vacuum approximation.

\section{References}

[1] Stringer T.E. Nucl. Fusion 12 (1972) 689

[2] Goldstone R.J., White R.B. and Boozer A.H. Phys. Rev. Lett. 47 (1981) 647

[3] Goldstone R.J. and Towner H.H. J. Plasma Phys. (1982) 26283

[4] K. Tani, T. Takizuka, M. Azumi and H. Kishimoto, Nucl. Fusion 23 (1983) 657

[5] K.Shinohara et al Fusion Eng. Des. (2009) 8424

[6] S.P.Hirshman and H.K.Meier, Phys. Fluids 28 (1985) 1387

[7] S.P.Hirshman, W.I.van Rij and P.Merkel, Comput. Phys. Comm. 43 (1986) 143

[8] Y.Suzuki et al. Nucl. Fusion 43 (2003) 406415

[9] E.Strumberger et al. Nucl. Fusion 50 (2010) 025008

[10] K.Shinohara et al. Nucl. Fusion 51 (2011) 063028

[11] Y.Nakamura et al. AIP Conf. Proc. 311 (1994) 114123

[12] R.H.Fowler, J.A.Rome and J.F.Lyon, Phys. Fluids 28 (1981) 338

[13] A.H.Boozer, Phys.Fluids 23 (1980) 904

[14] Gioietta Kuo-Petravic et al. J. comp. Phys. 51 (1983) 261

[15] H.Urano et al. Nucl. Fusion 47 (2007) 706

[16] O.A.Anderson and H.P.Furth, Nucl. Fusion 12207 (1972)

[17] K.Tobita et al. J. Plasma Fusion Res. 75582 (1999)

[18] M.Yokoyama, Y.Nakamura et al. Nucl. Fusion 40 (2000) 261

[19] K.Tani et al. J. Phys. Soc. Japan. 50 (1981) 1726

\section{Acknowledgments}

The authors thank S.P. Hirshiman and D.A. Spong for providing the VMEC code and the equilibrium data. This work was performed with the support and under the auspices of the NIFS Collaborative Research Program NIFS10KNTT004. This work was partially supported by a Grant-in-Aid for Scientific Research for Young Scientists (B) 20760585 from the Ministry of Education, Culture, Sports, Science and Technology. 